Pacific Journal of Mathematic 


\title{
ON GRAPHICAL REGULAR REPRESENTATIONS OF CYCLIC EXTENSIONS OF GROUPS
}

\author{
WILFRIED IMRICH AND MARK E. WATKINS
}

A simple graph $X$ is said to be a graphical regular representation (GRR) of an abstract group $G$ if the automorphism group of $X$ is a regular permutation group and is isomorphic to $G$. If a group $G_{1}$ is a cyclic extension of a group $G$ which admits a GRR, the question is posed whether $G_{1}$ also admits a GRR. Nowitz and Watkins have given an affirmative answer if $G_{1}$ is non-abelian and finite and the index $\left[G_{1}: G\right] \geqq 5$. This paper applies some new graph theoretical techniques to investigate the problem if $\left[G_{1}: G\right]=2,3$ or 4 , whether or not $G_{1}$ is finite. As long as $G_{1}$ is non-abelian, an affirmative answer can again be given except in only finitely many unresolved cases.

1. Introduction. A simple graph $X$ with vertex set $V(X)$ and automorphism group $A(X)$ is a graphical regular representation $(G R R)$ of the group $G$ if (i) $G \cong A(X)$ and (ii) $A(X)$ acts on $V(X)$ as a regular permutation group; that is, given $u, v \in V(X)$, there exists a unique $\varphi \in A(X)$ such that $\varphi(u)=v$. The graph $X$ is a $G R R$ if it is a GRR of some group.

A number of authors have investigated the question as to which finite groups $G$ admit a GRR. A complete and easily stated solution is known in case $G$ is abelian (see [10], [1], and [4]). If $G$ is non-abelian, the problem is more difficult and the results that have emerged to date seem to fall far less readily into a neat pattern, (see [6], [11], [7], [8], [12], and [13]). Nonetheless certain general principles appear to take form. For example, we are tempted to conjecture that if $G$ admits a GRR and $G \triangleleft G_{1}$ where $G$ is non-abelian, then $G_{1}$ admits a GRR. In particular, it has been shown [7, Theorem 1] that:

If $G$ admits a GRR and if the non-abelian group $G_{1}$ is a cyclic extension of $G$ such that the index $\left[G_{1}: G\right] \geqq 5$, then $G_{1}$ admits a GRR.

The techniques of [7] are inadequate, however, if $\left[G_{1}: G\right]=2,3$ or 4 , which is the task of the present paper. Elements of orders 2 and 3 have generally posed extra difficulties in the work on this problem. Thus [8, Theorem 2]:

Every non-abelian finite group whose order is coprime to 6 admits a GRR. 
Only affirmative answers are obtained for the existence of a GRR if $\left[G_{1}: G\right]=2,3$ or 4 . While the results are not complete, as in [7] and [8], the number of unresolved cases is finite, the results being "complete" if $|G|$ is sufficiently large. This fits in with another emerging principle that the only "large" groups not admitting a GRR are either abelian or generalized dicyclic (see [6] and [11]).

In $\$ 2$ notation will be introduced. The main result of the section (Theorem 1) is a graph theoretical result that bypasses the obstacles due to elements of orders 2 and 3 encountered in [7].

In $\$ 3$ existence of a GRR is demonstrated when $G$ (a group with a GRR) is extended to a non-abelian group by the cyclic groups $C_{2}$ and $C_{4}$, provided $G$ satisfies any of a variety of conditions. One such sufficient condition is simply that $|G|>32$.

In $\S 4$ the group $G$ is extended by $C_{3}$. The existence of a GRR follows when $|G|>36$ as well as when $|G|$ assumes certain smaller values and satisfies certain conditions.

2. Preliminaries. The letter $X$ will always denote a finite simple graph (except as noted in Corollary 1A below, where $X$ is infinite) with vertex set $V(X)$, edge set $E(X)$, and automorphism group $A(X)$. The complementary graph of $X$ relative to the complete graph on $|V(X)|$ vertices will be denoted by $X^{\prime}$. Thus $A(X)$ and $A\left(X^{\prime}\right)$ are identical, even as permutation groups. If $u \in V(X)$, the stabilizer of $u$ is the subgroup

$$
A_{u}(X)=\{\varnothing \in A(X) \mid \varnothing(u)=u\} .
$$

The valence (degree) of the vertex $u$ is denoted $\rho(u)$. The graph $X$ is said to be $\rho$-valent if $\rho(u) \equiv \rho$ for all $u \in V(X)$, and $X$ is isovalent if $X$ is $\rho$-valent for some cardinal $\rho$. (We depart here from the more conventional term "regular", which serves in another capacity in this paper.)

The letter $G$ (with or without a subscript) will always denote a finite group. Its automorphism group will be denoted by Aut $(G)$, its center by $Z(G)$, and its identity element by $e$. The cyclic group of order $n$ will be denoted by $C_{n}$ and the dihedral group of order $2 n$ by $D_{n}$. If $K$ is a subset of $G$, then $\langle K\rangle$ represents the subgroup of $G$ generated by $K$. If $g \in G$, the order of $g$ is $o(g)=|\langle g\rangle|$. The exponent of $G$, abbreviated by $\exp (G)$, is the least common multiple of the orders of the elements of $G$.

The letter $H$ (with or without a subscript) will always denote a subset of the group $G$ (bearing the same subscript, if any) with the following three properties: (i) $e \notin H$, (ii) $h \in H \Rightarrow h^{-1} \in H$, (iii) $\langle H\rangle=G$.

The Cayley graph $X_{G, H}$ of $G$ with respect to $H$ is the graph with $V\left(X_{G, H}\right)=G$, and $[x, y] \in E\left(X_{G, H}\right)$ if and only if $y=x h$ for some $h \epsilon$ 
H. A Cayley graph $X=X_{G, H}$ is always connected, and since $A(X)$ contains all left-multiplications by elements of $G$, it is transitive on $V(X)$. If $X$ is a GRR of $G$ and $|G|>2$, then $X$ must be a Cayley graph $X_{G, H}$ for some $H$, (see [9, Theorem 2]). Moreover, if $X$ is a GRR of $G$, then so is $X^{\prime}$, and $X$ is $\rho$-valent with $3 \leqq \rho \leqq|V(X)|-4$, (see [13, Lemma 1]).

The group $G$ is in Class $I$ if $G$ admits a GRR. It is in Class II if for each $H$ there exists $\varphi \in \operatorname{Aut}(G)$ such that $\varphi[H]=H$ but $\varphi$ is not the identity. These two classes are disjoint [11, Theorem 1], and it has been conjectured that every finite group is in one of these two classes.

To prove that a given Cayley graph $X=X_{G, H}$ is a GRR of $G$, it suffices to show (see [7, Corollary 2.4]) that for some set $K \subset H$ such that $\langle K\rangle=G$,

$$
\varphi \in A_{e}(X), h \in K \Longrightarrow \varphi(h)=h .
$$

The only abelian groups in Class I are the elementary abelian 2-groups $\left(C_{2}\right)^{n}$ for $n \neq 2,3,4$. (See [4] and [10].)

For integers $n \geqq 2, k \geqq 1$, a graph $X$ will belong to the class $\mathscr{P}_{n, k}$ if it is isovalent and if there exists a partition $\left[V_{1}, V_{2}, \cdots, V_{p}\right]$ of $V(X)$ with $2 \leqq p \leqq n$ such that every $x \in V_{i}$ is adjacent to $\leqq k$ elements of $V_{j},(i \neq j)$. Such a partition $\left[V_{1}, \cdots, V_{p}\right]$ is called a $\mathscr{P}_{n, k^{-}}$ partition. We also allow $\left[V_{1}, \cdots, V_{p}\right]$ to denote a $\mathscr{P}_{n, k}$-partition when as many as $p-2$ cells are empty.

It is an immediate consequence of these definitions that if $\left[V_{1}\right.$, $\left.\cdots, V_{p}\right]$ is a $\mathscr{P}_{n, k}$-partition of $X$ and if a vertex $u$ of $X$ is adjacent to $>k$ elements of $V_{i}$, then $u \in V_{i}$. Also, if $n \leqq n^{\prime}$ and $k \leqq k^{\prime}$, then $\mathscr{P}_{n, k} \subset \mathscr{P}_{n^{\prime}, k^{\prime}}$.

TheOREm 1. Let $X \in \mathscr{P}_{n, k}$ and suppose $|V(X)|>n(m h+2 k)$, where $m, h$ are integers such that $m \geqq 2, h \geqq 1$. Then $X^{\prime} \notin \mathscr{P}_{m, h}$.

Proof. Suppose $X$ satisfies the hypothesis of the theorem but not the conclusion. Let $\left[V_{1}, \cdots, V_{p}\right]$ be a $\mathscr{P}_{n, k}$-partition of $V(X)$ where the cells have been indexed so that

$$
\left|V_{i}\right| \geqq\left|V_{i+1}\right| \quad(i=1, \cdots, p-1) .
$$

Then $n\left|V_{1}\right| \geqq p\left|V_{1}\right| \geqq|V(X)|>n(m h+2 k)$, and so

$$
\left|V_{1}\right|>m h+2 k \text {. }
$$

Arbitrarily choose and fix $a \in V(X) \backslash V_{1}$, and let

$$
N_{1}=\left\{x \mid x \in V_{1},[a, x] \in E(X)\right\} .
$$


Then $\left|N_{1}\right| \leqq k$, since $X \in \mathscr{P}_{n, k}$.

By assumption there exists a $\mathscr{P}_{m, h}$-partition $\left[W_{1}, \cdots, W_{q}\right]$ of $X^{\prime}$. Define $U_{i}=W_{i} \cap\left(V_{1} \backslash N_{1}\right)$. Without loss of generality we may assume that $\left|U_{1}\right| \geqq\left|U_{i}\right|(i=2, \cdots, q)$.

By (2.1), $m h+k<\left|V_{1}\right|=\left|N_{1}\right|+\sum_{i=1}^{q}\left|U_{i}\right| \leqq k+m\left|U_{1}\right|$, and so $h<\left|U_{1}\right|$. Since the vertex $a$ is adjacent in $X^{\prime}$ to all vertices of $U_{1}$, we have $a \in W_{1}$. Moreover, $a$ is adjacent to $\leqq h$ vertices in each cell $W_{i}(i=2, \cdots, q)$. Hence $\left|U_{i}\right| \leqq h$, and so $\left|N_{1}\right|+\sum_{i=1}^{q}\left|U_{i}\right| \leqq$ $k+\left|U_{1}\right|+(m-1) h$. By (2.1), $\left|U_{1}\right|>h+k$. Since each vertex in $V(X) \backslash V_{1}$, is adjacent to $\leqq k$ and nonadjacent to $>h$ vertices in $U_{1}$,

$$
V(X) \backslash V_{1} \subset W_{1}
$$

Arbitrarily choose and fix $c \in V_{1} \backslash U_{1}$. It will be shown that $c \in W_{1}$, which together with (2.2) implies that $W_{1}=V(X)$, contrary to the assumption that $\left[W_{1}, \cdots, W_{q}\right]$ is nontrivial, and the theorem will follow.

Suppose $c \notin W_{1}$. By (2.2), $c \in V_{1}$ and so $c$ is adjacent in $X$ to $\leqq(p-1) k$ vertices of $V(X) \backslash V_{1}$. Hence $c$ is adjacent in $X^{\prime}$ to $\geqq\left|V(X) \backslash V_{1}\right|-(p-1) k$ vertices of $V(X) \backslash V_{1}$. But $c$ is adjacent in $X^{\prime}$ to $\leqq h$ vertices of $W_{1}$. Hence $h \geqq\left|V(X) \backslash V_{1}\right|-(p-1) k$. This implies $h \geqq(p-1)\left(\left|V_{p}\right|-k\right)$, whence $h+k \geqq\left|V_{p}\right|$, since $p-1 \geqq 1$.

Suppose $X$ is $\rho$-valent. Then by consideration of a vertex in $V_{p}$ it is easily seen that

$$
\rho \leqq(p-1) k+\left|V_{p}\right|-1 \leqq p k+h-1 .
$$

Also, in the light of (2.2),

$$
\left|V(X) \backslash W_{1}\right| \leqq\left|N_{1}\right|+\sum_{i=2}^{q}\left|U_{i}\right| \leqq k+(m-1) h .
$$

Now $c$ is adjacent in $X$ to $\leqq \rho$ vertices in $W_{1}$. Hence by (2.1), (2.3), and (2.4), the number of vertices of $W_{1}$ to which $c$ is adjacent in $X^{\prime}$ is at least

$$
\begin{aligned}
\left|W_{1}\right|-\rho & =|V(X)|-\left|V(X) \backslash W_{1}\right|-\rho \\
& \geqq n(m h+2 k)-[k+(m-1) h]-(p k+h-1) \\
& =(n-1) m h+(2 n-p-1) k+1>h,
\end{aligned}
$$

which implies that $c \in W_{1}$.

Corollary 1A. Let $X \in \mathscr{P}_{n, k}$ be a infinite graph. Then $X^{\prime}$ belongs to no class $\mathscr{P}_{m, h}$.

Proof. Let $X$ be infinite and let $\left[V_{1}, \cdots, V_{p}\right]$ be a $\mathscr{P}_{n, k}$-partition of $X$. Suppose $X^{\prime} \in \mathscr{P}_{m, h}$ and let $\left[W_{1}, \cdots, W_{q}\right]$ be a $\mathscr{P}_{m, h}$-partition of $X^{\prime}$. Without loss of generality, we can assume that $V_{1} \cap W_{1}$ is 
infinite. Since each vertex in $V(X) \backslash V_{1}$ is adjacent in $X$ to $\leqq k$ vertices of $V_{1} \cap W_{1}$, it is adjacent in $X^{\prime}$ to infinitely many vertices of $V_{1} \cap W_{1}$ and is therefore in $W_{1}$. Thus $V(X) \backslash V_{1} \subset W_{1}$.

If $V_{i}$ were infinite for some $i \neq 1$, then each vertex of $V_{1}$-and hence each vertex of $V(X) \backslash W_{1}$-would be adjacent in $X^{\prime}$ to infinitely many vertices of $V_{i}$. That is to say, each vertex in each $W_{j}(j \neq 1)$ would be adjacent in $X^{\prime}$ to infinitely many vertices of $W_{1}$, contrary to assumption. Hence $V_{2}, \cdots, V_{p}$ are each finite sets. Consideration of a vertex, say in $V_{p}$, yields that $X$ is $\rho$-valent for some finite cardinal $\rho$. But then the valence $\rho^{\prime}$ of $X^{\prime}$ is infinite. A vertex in, say, $W_{q}$ is therefore adjacent in $X^{\prime}$ to infinitely many vertices of $W_{q}$ which is absurd, for $W_{q}$ is finite since each vertex of $W_{1}$ is adjacent to $\leqq \rho$ vertices of $W_{q}$ and nonadjacent to $\leqq h$ vertices of $W_{q}$.

For integers $n \geqq 2, k \geqq 1$, define $\nu(n, k)$ to be the smallest integer for which it holds that whenever $X \in \mathscr{P}_{n, k}, X$ is a GRR, and $|V(X)|>$ $\nu(n, k)$, then $X^{\prime} \notin \mathscr{P}_{n, k}$. The following is immediate.

COROLlary 1B. If $n \geqq 2$ and $k \geqq 1$, then

$$
\nu(n, k) \leqq k n(n+2) \text {. }
$$

The inequality in Corollary $1 \mathrm{~B}$ is not necessarily as strong as possible. While the authors have no uniform procedure for determining $\nu(n, k)$ in general, improvements over the corollary exist in special cases. For example, Corollary $1 \mathrm{~B}$ gives only $\nu(3,1) \leqq$ 15 and $\nu(2,2) \leqq 16$. The following stronger results will be used in $\S \S 3$ and 4 .

LEMMA $1 . \quad \nu(3,1) \leqq 11$.

Proof. Let $\left[V_{1}, V_{2}, V_{3}\right]$ be a $\mathscr{P}_{3,1}$-partition of a GRR $X$ where $|V(X)| \geqq 12$, and suppose $\left|V_{1}\right| \geqq\left|V_{2}\right| \geqq\left|V_{3}\right|$. Clearly $\left|V_{1}\right| \geqq 4$. Note that $\rho \geqq 3$, since $X$ is a GRR. Supposing the corollary false, let [ $W_{1}$, $\left.W_{2}, W_{3}\right]$ be a $\mathscr{P}_{3,1}$-partition of $X^{\prime}$.

Suppose $\left|V_{1}\right|=4$ or 5 . Clearly then $\left|V_{2}\right| \geqq 4$ and $\left|V_{3}\right| \geqq 2$. Also $V_{1}$ must intersect some cell of $\left[W_{1}, W_{2}, W_{3}\right]$ in at least two vertices; say $a_{1}, a_{2} \in V_{1} \cap W_{1}$. With $k=1$, at least two vertices $b_{1}, b_{2} \in V_{2}$ are adjacent in $X$ to neither $a_{1}$ nor $a_{2}$ and so they are adjacent in $X^{\prime}$ to both $a_{1}$ and $a_{2}$. Hence $b_{1}, b_{2} \in W_{1}$. If $c \in V_{3}$, then $c$ is adjacent in $X$ to at most one of $a_{1}, a_{2}$ and at most one of $b_{1}, b_{2}$. So $c$ is adjacent in $X^{\prime}$ to at least two of these four vertices. Hence $c \in W_{1}$. We now have that 
Moreover, any vertex $d \in V_{i}$ is adjacent in $X^{\prime}$ to at least one vertex in $V_{j} \cap W_{1}$ for both values of $j \neq i$, whence $d \in W_{1}$. We infer the contradiction that $W_{1}=V(X)$.

Now suppose $\left|V_{1}\right| \geqq 6$. We assert that the inequality

$$
\left|V_{1} \cap W_{j}\right| \geqq 2
$$

can hold for at most two values of $j=1,2,3$. For if (2.5) held for $j=1,2,3$, then any vertex $b \in V_{2}$, being adjacent in $X$ to at most one vertex of $V_{1}$, is adjacent in $X^{\prime}$ to at least two vertices in each of two of the sets $V_{1} \cap W_{j}$, implying that $b$ is in two different cells of $\left[W_{1}, W_{2}, W_{3}\right]$. Since $\left|V_{1}\right| \geqq 6$, we may say for definiteness that $\mid V_{1} \cap$ $W_{1} \mid \geqq 3$.

If $c \in V_{2} \cup V_{3}$, then $c$ is adjacent in $X^{\prime}$ to at least two vertices in $V_{1} \cap W_{1}$, and so $c \in W_{1}$. Thus $V_{2} \cup V_{3} \subset W_{1}$.

Pick $a \in W_{2} \cup W_{3}$. Then $a$ is adjacent in $X$ to all but at most one vertex in $W_{1}$. But since $a \in V_{1}, a$ is adjacent to at most one vertex in each of $V_{2}$ and $V_{3}$. Hence $\left|V_{2}\right| \leqq 2$ and $\left|V_{3}\right| \leqq 1$. But $\left|V_{3}\right| \neq$ 1 , or else the lone vertex in $V_{3}$ would be adjacent in $X$ to two vertices in $V_{1}$ or in $V_{2}$ since $\rho \geqq 3$. Hence $V_{3}=\varnothing$. But then a vertex in $V_{2}$ would be adjacent (in $X$ ) to at least two vertices in $V_{1}$, giving a contradiction. It follows that $W_{2} \cup W_{3}=\varnothing$, and no $\mathscr{P}_{3,1}$-partition of $X^{\prime}$ exists.

LEMMA 2. $\nu(2,2) \leqq 15$.

Proof. Since $\nu(2,2) \leqq 16$ by Corollary $1 \mathrm{~B}$, it may be assumed that $X=X_{G, H}$ is a GRR where $|G|=16$. Let $\left[V_{1}, V_{2}\right]$ be a $\mathscr{P}_{2,2^{-}}$ partition of $X$, and suppose $\left[W_{1}, W_{2}\right]$ is a $\mathscr{P}_{2,2}$-partition of $X^{\prime}$. We show first that

$$
\left|V_{i} \cap W_{j}\right|=4, \quad i, j=1,2 .
$$

Supposing (2.6) false, we may assume without loss of generality that $\left|V_{1} \cap W_{1}\right| \geqq 5$. Then each vertex in $V(X) \backslash V_{1}$ is adjacent in $X^{\prime}$ to at least three vertices of $V_{1} \cap W_{1}$ and is therefore in $W_{1}$. Thus $V_{2} \subset W_{1}$.

If $\left|V_{2}\right| \geqq 5$ we conclude by similar argument that $V_{1}$ is also contained in $W_{1}$, which is not possible. If $\left|V_{2}\right| \leqq 4$, then $X$ is $\rho$-valent for $\rho \leqq 5$, and $\left|V_{1}\right| \geqq 12$. Now $\left|V_{1} \cap W_{2}\right| \leqq 4$ or else $V_{2} \subset W_{2}$, which is absurd. But then $\left|V_{1} \cap W_{1}\right| \geqq 8$ and, as every vertex in $W_{2}$ can be adjacent in $X$ to at most 5 vertices of $V_{1} \cap W_{1}$, it is adjacent in $X^{\prime}$ to at least three vertices in $V_{1} \cap W_{1}$ and hence is in $W_{1}$. This proves (2.6).

Each vertex $a \in V_{1} \cap W_{1}$ must be adjacent in $X$ to exactly two 
vertices in $V_{2} \cap W_{2} ; a$ cannot have more than two neighbors in $V_{2} \cap W_{2}$ since $X \in \mathscr{P}_{2,2}$, while if there were fewer than two, $a$ would be adjacent in $X^{\prime}$ to at least three vertices of $V_{2} \cap W_{2}$, in contradiction to the assumption that $a \in W_{1}$. Furthermore, $a$ must be adjacent in $X$ to all vertices in $V_{1} \cap W_{2}$. Otherwise $a$ would be adjacent in $X^{\prime}$ to at least one vertex in $V_{1} \cap W_{2}$ and to two vertices in $V_{2} \cap W_{2}$, making $a$ adjacent in $X^{\prime}$ to at least three vertices in $W_{2}$, which is not possible. In summary, with $i \neq i^{\prime}$ and $j \neq j^{\prime}$, each vertex in $V_{i} \cap W_{j}$ is adjacent to all vertices in $V_{i} \cap W_{j^{\prime}}$, to exactly two vertices in $V_{i^{\prime}} \cap W_{j^{\prime}}$, and to no vertices of $V_{i^{\prime}} \cap W_{j},\left(i, i^{\prime}, j, j^{\prime}=1,2\right)$.

Thus $\rho \geqq 6$. It may be assumed that $\rho \leqq 7$; otherwise $X$ and $X^{\prime}$ can be interchanged.

The eight edges joining $V_{1} \cap W_{1}$ with $V_{2} \cap W_{2}$ span a 2-valent subgraph on eight vertices which must be either the union of two disjoint 4-gons or a single 8-gon. In the first case let $\left[v_{1}, \cdots, v_{4}\right]$ and $\left[v_{5}, \cdots, v_{8}\right]$ denote the two 4-gons. In the second case let $\left[v_{1}, \cdots, v_{8}\right]$ denote the 8-gon. In either case, one may suppose that the vertices with odd index lie in $V_{1} \cap W_{1}$.

Let $T_{i}$ denote the subgraph of $X$ induced by the vertices in $V_{i} \cap$ $W_{i}(i=1,2)$. If $\rho=6$, then $T_{i}$ is totally disconnected. If $\rho=7$, then each $T_{i}$ consists of two edges without common end-point.

Consider the permutation $\beta$ on $V(X)$ whose cyclic decomposition is given by

$$
\beta=\left(v_{1} v_{5}\right)\left(v_{2} v_{6}\right)\left(v_{3} v_{7}\right)\left(v_{4} v_{8}\right)
$$

Since $\beta$ has fixed-points without being the identity, $\beta \notin A(X)$, for $A(X)$ is a regular permutation group. But clearly $\beta \in A(X)$ when $\rho=6$, and the verification is straightforward when $\rho=7$.

The result in Theorem 1 is far more general than is required for the applications in the next two sections. Actually, a rather narrow formulation is involved repeatedly. In the interest ultimately of economy, we here formulate that application in the following way:

Lemma 3. Let $G$ be a group in Class $I$. Let $n \geqq 2$ and $k \geqq 1$ be given and suppose $|G|>\nu(n, k)$. Then $G$ has a $G R R X=X_{G, H} \notin$ $\mathscr{P}_{n, k}$. Moreover, let $G \triangleleft G_{1}$ and suppose that for some $b \in G_{1} \mid G, G_{1}$ admits the coset decomposition $G_{1}=G \cup b G \cup \cdots \cup b^{n-1} G$. Let $H_{1}$ be a set of generators of $G_{1}$ such that

(i) $e \notin H_{1}=H_{1}^{-1}$,

(ii) $H_{1} \cap G=H$,

(iii) $k \geqq\left|H_{1} \cap b^{i} G\right|,(i=1, \cdots, n-1)$.

Form the Cayley graph $Y=X_{G_{1}, H_{1}}$, and let $X_{i}$ be the subgraph of $Y$ induced by vertices in the coset $b^{i} G$. Then $X_{i} \cong X$ and the sets 
$\left\{V\left(X_{i}\right) \mid i=0, \cdots, n-1\right\}$ form a complete system of imprimitivity for the permutation group $A(Y)$. Finally, if $\varphi \in A(Y)$ satisfies $\varphi x_{0}=$ $x_{0}$ for some $x_{0} \in V\left(X_{i}\right)$, then $\varphi x=x$ for all $x \in V\left(X_{i}\right)$.

Proof. If $X=X_{G, H}$ is a GRR of $G$, then so is $X^{\prime}$. Since $|V(X)|=$ $|G|>\nu(n, k)$, at most one of $X$ and $X^{\prime}$ belongs to $\mathscr{P}_{n, k}$ by definition of $\nu(n, k)$. For definiteness, suppose $X \notin \mathscr{P}_{n, k}$.

Assume the remainder of the hypothesis. That $X_{i} \cong X$ is immediate from the definition of a Cayley graph. Let $\varphi \in A(Y)$. Choose one of the subgraphs $X_{j}$ and let $V_{i}=V\left(X_{i}\right) \cap \varphi\left[V\left(X_{j}\right)\right]$. If $i \neq j$, each vertex of $X_{i}$ is adjacent to at most $k$ vertices of $X_{j}$. If more than one of the sets $V_{i}$ are nonempty, the sets $V_{i}$ form a $\mathscr{P}_{n, k^{-}}$ partition of $\varphi\left[X_{j}\right]$. But $\varphi\left[X_{j}\right] \cong X_{j} \cong X \notin \mathscr{P}_{n, k}$. Hence $\varphi\left[X_{j}\right]=X_{i}$ for some $i$. Since $Y$ is a Cayley graph, $A(Y)$ is transitive, and the assertion concerning imprimitivity is proved.

Now suppose $\varphi x_{0}=x_{0}$ for some $x_{0} \in V\left(X_{i}\right)$. Since $V\left(X_{i}\right)$ is a block of imprimitivity, $\varphi\left[X_{i}\right]=X_{i}$. Hence the restriction $\varphi_{i}$ of $\varphi$ to $X_{i}$ belongs to $A\left(X_{i}\right)$. But $X_{i} \cong X$ and so $A\left(X_{i}\right)$ is a regular permutation group. Since $\varphi_{i}$ has a fixed-point $x_{0}, \varphi_{i}$ is the identity of $A\left(X_{i}\right)$, which proves the lemma.

LEMma 4. Every non-abelian group $G$ of square-free order $|G|>$ 10 is Class $I$.

Proof. Since $|G|$ is square-free, $G$ is generated by two elements $a$ and $b$ satisfying relations of the form

$$
\left\langle a, b \mid a^{r}=b^{s}=e, b^{-1} a b=a^{m}\right\rangle
$$

where $|G|=r s$ and $m^{s} \equiv 1(\bmod r) . \quad($ See $[5$, p. 261].) All groups (2.7) have been classified [7, Theorem 2]. Except for the groups $D_{3}$ and $D_{5}$, the only groups (2.7) not in Class I are either abelian or have order divisible by 4 .

3. Classification of Extensions by $C_{2}$ and $C_{4}$. The main result of this section is:

THEOREM 2. Let the non-abelian group $G_{1}$ contain a subgroup $G$ of index 2, and suppose that $G$ is in Class $I$. Then each of the following conditions is sufficient for $G_{1}$ to be in Class $I$ :

(a) $|G|>2$ and $G_{1}$ is a semi-direct product of $G$ by $C_{2}$.

(b) $|G|>12$ but $|G| \neq 16$ or 32 .

(c) $|G|=16$ or 32 , and either:

(i) there exists an element $b \in G_{1} \backslash G$ such that $o(b) \neq 4$, or

(ii) $Z(G)$ does not contain $C_{2} \times C_{2}$ as a subgroup. 
REMARK. The reader should not infer that condition (c) is necessary if $|G|=16$ or 32 , but merely that the profusion of groups of orders 16 and 32 not satisfying (ii) and of their cyclic extensions not satisfying (i) renders a case-by-case study inordinately tedious and without anticipation of surprising outcome. Theorem 2 is stated in the given form merely to convey as much information as possible.

Groups of order 16 have been classified (see [12]) and exactly two such groups both are in Class I and fail to satisfy (ii). There are six groups $G_{1}$ of order 32 failing to satisfy (i) which are extensions of these two exceptional groups of order 16. There are more than 100 groups $G_{1}$ of order 64 failing to satisfy (i) which are extensions of those groups $G$ of order 32 failing to satisfy (ii). For a complete list of such groups, see [3].

Proof. First consider the case where there exists an element $b \in G_{1} \mid G$ of order 2. Since $G$ is in Class I and $G \not C_{2}$, we have $|G| \geqq$ 12 (see [7, 1.10]). By Corollary $1 \mathrm{~B}$ and Lemma $3, G$ has a GRR $X=$ $X_{G, H} \notin \mathscr{P}_{2,1}$. If $H_{1}=H \cup\{b\}$, one may form the Cayley graph $Y_{0}=$ $X_{G_{1}, H_{1}}$. Let $\varphi \in A_{e}\left(Y_{0}\right)$. With $X_{0}$ and $X_{1}$ defined as in Lemma 3 , it is immediate that $\varphi$ fixes each vertex of $X_{0}$. Hence $\varphi(b)=b$, and $\varphi$ is the identity on all of $V\left(Y_{0}\right)$, whence $Y_{0}$ is a GRR of $G_{1}$. It has been shown that (a) is a sufficient condition.

Suppose now that $b^{2} \neq e$ for all $b \in G_{1} \mid G$. If $|G|=14$, then $G \cong$ $D_{7}$. Of the two non-abelian groups of order 28 [2, p. 135], only one contains $D_{7}$ as a subgroup, namely $D_{14}$ which is known to be in Class I, [11, Theorem 2]. Since no group in Class I has order 13 or 15 we now let $|G| \geqq 16$. By Lemmas 2 and $3, G$ has a GRR $X=X_{G, H} \in \mathscr{P}_{2,2}$, as before. Arbitrarily choose then fix $b \in G_{1} \mid G$. Let $H_{1}=H \cup\left\{b, b^{-1}\right\}$, and form $Y_{1}=X_{G_{1}, H_{1}}$. Let $\varphi \in A_{e}\left(Y_{1}\right)$. As before, $\varphi$ fixes each vertex of $X_{0}$, and the theorem is proved unless $\varphi$ has no fixed-point in $X_{1}$, which we now assume to be the case. In particular, $\varphi$ interchanges $b$ and $b^{-1}$.

The vertices in $X_{0}$ adjacent to $b$ are $e$ and $b^{2}$ while those in $X_{0}$ adjacent to $b^{-1}$ are $e$ and $b^{-2}$. Thus $b^{2}=b^{-2}$, and $b^{4}=e$. Since the choice of $b \in G_{1} \mid G$ was arbitrary, it follows that $o(b)=4$ for all $b \in$ $G_{1} \mid G$.

We next show that $b^{2} \in Z\left(G_{1}\right)$ for all $b \in G_{1} \backslash G$. Let $h \in H$. Since $b^{-1}$ is adjacent to $b^{-1} h, b=\varphi\left(b^{-1}\right)$ must be adjacent to $\varphi\left(b^{-1} h\right)$. The neighbors in $X_{1}$ of $b^{-1} h b \in V\left(X_{0}\right)$ are $b^{-1} h$ and $b^{-1} h b^{2}$. Thus $\varphi\left(b^{-1} h\right)=$ $b^{-1} h b^{2}$. There exists some $h^{\prime} \in H$ such that $b h^{\prime}=b^{-1} h b^{2}$, i.e., $h^{\prime}=b^{-2} h b^{2}$. This implies that the set $H$ is fixed under conjugation by $b^{2}$, but since $X$ is a GRR, the automorphism $x \mapsto b^{-2} x b^{2}$ in Aut $(G)$ must be the identity. Thus $b^{2} \in Z(G)$. But for any $x \in G,(b x) b^{2}=b\left(b^{2} x\right)=b^{2}(b x)$, 
so $b^{2} \in Z\left(G_{1}\right)$, as required.

Recalling that the choice of $b \in G_{1} \mid G$ was arbitrary, we have that the set

$$
K=\left\{c^{2}\left|c \in G_{1}\right| G\right\}
$$

generates an elementary abelian 2-group contained in $Z\left(G_{1}\right) \cap G$. If $K$ consists of a single element, then $b^{2}=(b x)^{2}$ for all $x \in G, b \in G_{1} \mid G$. This implies that

$$
b^{-1} x b=x^{-1} ; \quad x \in G, b \in G_{1} \mid G .
$$

Thus $G$ is abelian. Since the only abelian groups in Class I are elementary abelian 2-groups, (3.1) implies that $G_{1}$ is abelian, contrary to the hypothesis of this theorem. Hence

$$
|K| \geqq 2 \text {. }
$$

At this point condition (c) has been shown sufficient.

Having obtained the foregoing information about $G_{1}$, we continue to prove the sufficiency of (b) by first supposing that $|G|>32$. By Corollary $1 \mathrm{~B}$ and Lemma 3 we may assume that $X=X_{G, H} \notin \mathscr{P}_{2,4}$. By (3.2), there exist $b, c \in G_{1} \mid G$ such that $b^{2} \neq c^{2}$. Redefine $H_{1}=H \cup$ $\left\{b, b^{-1}, c, c^{-1}\right\}$, and form $Y_{2}=X_{G_{1}, H_{1}}$. As before, let $\varphi \in A_{e}\left(Y_{2}\right)$. Again, $\varphi$ fixes each vertex of $X_{0}$, and we may assume that $\phi$ fixes no vertex of $X_{1}$. For each $x \in V\left(X_{0}\right)$, its neighbors in $X_{1}$ are $x b, x b^{-1}, x c, x c^{-1}$ while the neighbors in $X_{1}$ of $x b^{2}$ are $x b^{-1}, x b, x b^{2} c, x b^{2} c^{-1}$. Since $b^{2} \neq$ $c^{2}$ (and so $b \neq c^{-1}$ ), the vertices $x$ and $x b^{2}$ have precisely two common neighbors in $X_{1}$, namely $x b$ and $x b^{-1}$. They must be interchanged by $\varphi$, as are $x c$ with $x c^{-1}$. This is so for all $x \in V\left(X_{0}\right)$. In particular, for $x=e$ or $b^{-1} c$ we obtain

$$
b=\varphi\left(b^{-1}\right)=\varphi\left(\left(b^{-1} c\right) c^{-1}\right)=b^{-1} c^{2},
$$

implying that $b^{2}=c^{2}$, a contradiction. Hence $Y_{2}$ is a GRR of $G_{1}$.

It remains only to consider the cases where $16<|G|<32$. Because of (3.2), $G$ contains the central subgroup $M=\langle K\rangle$ of order divisible by 4 , leaving only 20,24 , and 28 as possible orders of $G$. If $|G|=$ 20 or 28 , then $G / M$ is cyclic. But then $G$ is abelian but not an elementary abelian 2-group, contrary to the hypothesis that $G$ is in Class I.

Suppose finally that $|G|=24$. It suffices to prove that $G$ is abelian, for then it cannot be in Class I. There exists an element $a \in G$ of order 3. We show first that

$$
a^{-1}=d^{-1} a d \equiv a^{d}
$$

for all $d \in b G$.

Since $d^{2},(d a)^{2} \in K$, one has immediately that 


$$
d^{2} a a^{d}=a d^{2} a^{d}=(a d)^{2}=d^{-1}(d a)^{2} d=(d a)^{2}=d^{2} d^{-1} a d a=d^{2} a^{d} a .
$$

It follows that $a$ and $a^{d}$ commute and that $\left(a a^{d}\right)^{2}=\left[d^{-2}(d a)^{2}\right]^{2}=$ $d^{-4}(d a)^{4}=e$. Since $o\left(a^{d}\right)=3$,

$$
a a^{d}=\left(a a^{d}\right)^{2} a a^{d}=a^{3}\left(a^{d}\right)^{3}=e,
$$

and hence (3.3) holds. Now write $d=x b$ for arbitrary $x \in G$. By (3.3), for any $x \in G$,

$$
a x b=(x b) a^{-1}=x\left(b a^{-1}\right)=x a b .
$$

This shows that $a \in Z(G)$. Therefore, the subgroup $\langle M \cup\{a\}\rangle$ is central and has order 12 , which is possible only if $G$ is abelian. This concludes the proof of the theorem.

Remarks. 1. Except in the case where $G$ is abelian, Theorem 2 generalizes the result of Watkins and Nowitz [13] that the direct product of $C_{2}$ with any group in Class I other than $C_{2}$ is also in Class I.

2. In addition to the special cases of groups of orders 16 and 32 previously remarked upon, the question remains open concerning the classification of extensions by $C_{2}$ (other than semi-direct products) of the one group in Class I of order 12, namely $D_{6}$.

COROLLARY 2A. Let the non-abelian group $G_{1}$ contain a normal subgroup $G$ such that $G_{1} / G \cong C_{4}$, and suppose that $G$ is in Class $I$. If $|G|>32$, then $G_{1}$ is in Class $I$.

Proof. Let $\pi$ be the projection morphism of $G_{1}$ onto $G_{1} / G \cong C_{4}=$ $\langle a\rangle$. If $\pi(b)=a$, we have the coset decomposition

$$
G_{1}=G \cup b G \cup b^{2} G \cup b^{3} G .
$$

Furthermore, $G_{0}=G \cup b^{2} G$ is a subgroup of $G_{1}$ and $\left[G_{0}: G\right]=\left[G_{1}: G_{0}\right]=$ 2. If $G_{0}$ is non-abelian, it is in Class I by Theorem 2, and therefore so is $G_{1}$.

We have only to consider the case where $G_{0}$ is abelian. Then $G$ is abelian, and since $G$ is in Class $I$, we have $G \cong\left(C_{2}\right)^{k+1}$ with $k \geqq 5$ because $|G|>32$. If $b^{4}=e$, the group $G_{0}$ is an elementary abelian 2-group and so is in Class I. Then $G_{1}$ is also in Class I by Theorem 2. We can therefore assume that $b^{4} \neq e$. As $b^{4} \in G$ and $\exp (G)=2$, we have $o(b)=8$.

Denote by $K$ the subgroup of $G$ such that $G=K \times\left\langle b^{4}\right\rangle$. Note that $K \cong\left(C_{2}\right)^{k}$ is in Class I since $k \geqq 5$. If $K \triangleleft G_{1}$, then $G_{1}$ is an extension of $K$ by $C_{8}$ and is in Class I by [7, Theorem 1]. Otherwise $b K \neq K b$, and there exists an element $c \in K$ such that $b c \neq c b$. Using 
this $c$ we shall construct a GRR of $G_{1}$.

Since $|G| \geqq 64>48 \geqq \nu(4,2), G$ admits a GRR $X=X_{G, H} \notin \mathscr{P}_{4,2}$, by Lemma 3 . The set

$$
H_{1}=H \cup\left\{b, b^{-1}, b^{2}, b^{-2}, b c, c b^{-1}\right\}
$$

generates $G_{1}$. We shall demonstrate that the Cayley graph $Y_{1}=X_{G_{1}, H_{1}}$ is a GRR.

First observe the distribution of elements of $H_{1} \backslash H$ amongst the cosets of $G$ :

$$
b, b c \in b G ; b^{2}, b^{-2} \in b^{2} G ; b^{-1}, c b^{-1} \in b^{3} G \text {. }
$$

Let $\varphi \in A_{e}\left(Y_{1}\right)$. By Lemma 3 (and in the same notation as in the lemma), $\varphi$ fixes each vertex of $X_{0}$. In particular, $\varphi$ fixes the four distinct vertices $b^{4}, b c b^{-1}, c$, and $e$. The only neighbors of $b$ and $b c$ in $X_{0}$ are $e$ and $b c b^{-1}$; the vertices $b^{2}$ and $b^{-2}$ have the neighbors $e$ and $b^{4} \in V\left(X_{0}\right)$; and $b^{-1}$ and $c b^{-1}$ are each adjacent to $e$ and $c \in V\left(X_{0}\right)$. Therefore, $\varphi$ fixes each $V\left(X_{i}\right)$ setwise. As $b$ is adjacent to $b^{2}$ but not to $b^{-2}$, while $b c$ is adjacent to neither $b^{2}$ nor $b^{-2}$, it is clear that $\varphi$ cannot interchange $b$ with $b c$. Hence $\varphi(b)=b$. As $\varphi$ fixes every element of the generating set $\{b\} \cup H$ of $G_{1}$, it fixes every element of $G_{1}$. This proves the corollary.

\section{Classification of extensions by $C_{3}$.}

THEOREM 3. Let the non-abelian group $G_{1}$ contain a normal subgroup $G$ of index 3, and suppose $G \not C_{2}$ is in Class I. Then each of the following conditions is sufficient for $G_{1}$ to be in Class I:

(a) There exists an element $b \in G_{1} \mid G$ such that $b^{6} \neq e$.

(b) $|G|>30$ and either:

(i) $G \not \equiv\left(C_{2}\right)^{5}$,

or

(ii) if $|G|=36$, then $\exp (G) \neq 6$.

(c) $G_{1}=G \times C_{3}$, and if $|G|=24$ or 36 , then $\exp (G) \neq 6$.

Proof. (a) By hypothesis, $|G| \geqq 12$. Lemmas 1 and 3 imply that $G$ has a GRR $X=X_{G, H} \notin \mathscr{P}_{3,1}$. Assuming condition (a), let $H_{1}=$ $H \cup\left\{b, b^{-1}\right\}$ and form $Y_{0}=X_{G_{1}, H_{1}}$. Let $\varphi \in A_{e}\left(Y_{0}\right)$. By Lemma 3 (and in the same notation as in the lemma), $\phi$ fixes each vertex of $X_{0}$. It suffices to prove that $\varphi$ fixes $b$ (and hence $b^{-1}$ ). We suppose not, and so $\varphi$ interchanges $b$ with $b^{-1}$. Lemma 3 further implies that $\varphi$ maps the subgraphs $X_{1}$ and $X_{-1}$ isomorphically onto each other.

Since the only neighbor of $b^{-1}$ lying in $X_{1}$ is $b^{-2}, \varphi\left(b^{-2}\right)$ must be the unique neighbor of $b=\varphi\left(b^{-1}\right)$ lying in $X_{-1}$, namely $b^{2}$. But $b^{3} \in$ 
$V\left(X_{0}\right)$ is fixed by $\varphi$, and so $\varphi$ interchanges its neighbors $b^{4} \in V\left(X_{1}\right)$ with $b^{2} \in V\left(X_{-1}\right)$. Thus $\varphi\left(b^{4}\right)=b^{2}=\varphi\left(b^{-2}\right)$, whence $b^{4}=b^{-2}$, or $b^{6}=e$, contrary to assumption.

(b) In the light of (a) above, it may be assumed that

$$
o(u)=3 \text { or } 6 \quad \text { for all } u \in G_{1} \mid G .
$$

If $o(u)=6$, then $o\left(u^{2}\right)=3$. So we may always select an element $d \in G_{1} \backslash G$ of order 3. Write the coset decomposition $G_{1}=G \cup d G \cup d^{-1} G$.

We shall now prove that:

$$
\exists b, c \in d G \text { such that } b^{3}=e \text { and } \quad b c \neq c b .
$$

If $G$ contains an element $g$ such that $g^{6} \neq e$, let $c=g b$, where $b \in d G$ has order 3 . If $b$ commutes with $c$, then $b$ commutes with $g$, and

$$
c^{6}=(g b)^{6}=g^{6} b^{6}=g^{6} \neq e,
$$

contrary to (4.1).

Suppose then that $g^{6}=e$ for all $g \in G$ and that (4.2) fails. Since $d^{3}=e, d$ commutes with every element of $G_{1} \backslash G$. Thus, for all $g \in G$ $(d g) d=d(d g)$, and $d \in Z\left(G_{1}\right)$. Define

$$
K=\{x \in G \mid o(d x)=3\} .
$$

Then $x \in G$ implies $x^{3}=(d x)^{3}$, while $(d x)^{3}=e$ if and only if $x \in K$. Thus for $x \in G$,

$$
\begin{gathered}
x \in K \Longleftrightarrow o(x)=1 \text { or } 3 ; \\
x \in G \backslash K \Longleftrightarrow o(x)=2 \text { or } 6 .
\end{gathered}
$$

If $x \in K$ and $g \in G$, then since (4.2) is assumed false, $(d x)(d g)=(d g)(d x)$. Since $d$ is central we have $x g=g x$, and so $K \subset Z(G)$. If $x_{1}, x_{2} \in K$, then $\left(d x_{1} x_{2}\right)^{3}=d^{3} x_{1}^{3} x_{2}^{3}=e$, so $x_{1} x_{2} \in K$, and $K$ is a normal subgroup of $G$.

Consider the cosets of $G$ with respect to $K$. Since by (4.3) and (4.4) $x^{2} \in K$ for all $x \in G$, the quotient group $G / K$ is an elementary abelian 2-group. Moreover, if $x_{1}=x_{2} k$ for some $x_{1}, x_{2} \in G$ and $k \in K$, then

$$
x_{1} x_{2}=x_{2} k x_{2}=x_{2}\left(x_{2} k\right)=x_{2} x_{1} .
$$

That is to say, any two elements of the same coset with respect to $K$ commute.

Let $L=\left\{x \in G \mid x^{2}=e\right\}$, and let $y, z \in L$. If $o(y z)=3$ or 6 , then $o\left((y z)^{2}\right)=3$ whence $(y z)^{2} \in K$ and is therefore central. Hence

$$
e=(y z y z) y^{2}(z y z y)=(y z)^{2} y(y z)^{2} y=(y z)^{4} y^{2}=(y z)^{4} .
$$


As no element of $G$ has order $4,(y z)^{2}=e$ and $y z \in L$. Hence $L$ is a subgroup of $G$. In particular, $L$ is an elementary abelian 2-group.

Let $g \in G$. If $o(g)=1,2$ or 3 , clearly $g \in L K$. If $o(g)=6$, then $g=g^{3} g^{-2} \in L K$, and so $G=L K$. Since $K$ is central and $L$ is abelian, $G$ is abelian. But $G_{1}=\langle G \cup\{d\}\rangle$. We have a contradiction, since $G_{1}$ is non-abelian by hypothesis. This proves (4.2).

We now assume the hypothesis (b). By Corollary $1 \mathrm{~B}$ and Lemma 3, $G$ has a GRR $X=X_{G, H} \notin \mathscr{P}_{3,2}$ Select $b, c$ fulfilling (4.2). Let $H_{1}=$ $H \cup\left\{b, b^{-1}, c, c^{-1}\right\}$ and form the Cayley graph $Y_{1}=X_{G_{1}, H_{1}}$. Let $\varphi \in$ $A_{e}\left(Y_{1}\right)$. As in all the previous cases, $\varphi$ fixes each vertex of $X_{0}$, in particular $b c^{-1}$.

The neighbors in $X_{-1}$ of $e$ are of course $b^{-1}$ and $c^{-1}$; those of $b c^{-1}$ are $b c^{-1} b^{-1}$ and $b c^{-2}$. Since $b \neq c$, certainly $b^{-1} \neq b c^{-1} b^{-1}$ and $c^{-1} \neq b c^{-2}$. If $c^{-1}=b c^{-1} b^{-1}$, then $b c=c b$, contrary to assumption. Also the equality $b^{-1}=b c^{-2}$ implies, since $b^{3}=e$, that $b=c^{-2}$ which in turn implies that $b$ and $c$ commute. Hence $e$ and $b c^{-1}$ have no common neighbors in $X_{-1}$.

The vertex $b \in V\left(X_{1}\right)$ is a common neighbor of $e$ and $b c^{-1}$. Thus $\varphi(b) \in V\left(X_{1}\right)$, and by Lemma $3, \varphi\left[X_{\imath}\right]=X_{i}(i= \pm 1)$. If $b$ is the only such common neighbor, then $\varphi(b)=b, \varphi$ is necessarily the identity, and (b) is proved. Suppose that $b$ is not the sole common neighbor. This is possible only if $c=\left(b c^{-1}\right) b$, which implies

$$
\left(c b^{-1}\right)^{2}=e,
$$

which we assume to hold.

If $G$ contains an element $g$ such that $g^{6} \neq e$, then, as previously noted, we may choose $c=g b$. Substitution into (4.5), however, implies that $g^{2}=e$. Assume that $g^{6}=e$ for all $g \in G$. Inasmuch as the group $\left(C_{2}\right)^{5}$ and all groups of order 36 with exponent 6 are exempted from consideration, no cases are lost if one assumes that $|G|>45$. Thus it may be assumed that $X \notin \mathscr{P}_{3,3}$. In addition to $b, c \in d G$ chosen according to (4.2), we select an additional element $f \in d G$ such that

$$
f \in\left\{b, b c b^{-1}, b c^{-1} b, b^{-1} c^{-1}, c, c b c^{-1}, c b^{-1} c, c^{2} b^{-1}\right\} .
$$

Redefine $H_{1}=H \cup\left\{b, b^{-1}, c, c^{-1}, f, f^{-1}\right\}$ and form $Y_{2}=X_{G_{1}, H_{1}}$. One can verify straightforwardly that despite the additional edges, $b c^{-1}$ and $e$ still have no common neighbors in $X_{-1}$ and that $b$ and $c$ remain their only common neighbors in $X_{1}$. Since $f$ is the only neighbor in $X_{1}$ of $e$ which is not a neighbor of $b c^{-1}, \varphi(f)=f$. Thus $\varphi$ fixes pointwise the set $H \cup\{f\}$ which generates $G_{1}$. Hence $\phi$ is the identity and $Y_{2}$ is a GRR of $G$.

(c) Let $G_{1}=G \times C_{3}$. We first suppose that $G$ contains an element $g$ such that $g^{6} \neq e$. There exists an element $c \in G_{1} \mid G$ such that $c^{3}=e$. 
If $b=g c$,

$$
b^{6}=(g c)^{6}=g^{6} c^{6}=g^{6} \neq e,
$$

and the result follows from part (a) above.

Suppose therefore, that $\exp (G)=2,3$ or 6 . By part (b), we need only assume $12 \leqq|G| \leqq 36$ and ignore the orders 24 and 36 . If $\exp (G)=$ 2 , then $G$ is abelian. Since $G_{1}$ is non-abelian, there are no cases to consider. If $\exp (G)=3$, then $|G|=27$. But the (unique) group $G$ of order 27 with exponent 3 is in Class II, [8, Theorem 3].

If $\exp (G)=6$, then $|G|=12$ or 18 .

Since $G$ is in Class I, $G \cong C_{3} \times D_{3}$ if $|G|=18$, and $G \cong D_{6}$ if $|G|=12$. (See [12].)

Following Coxeter and Moser [2, p. 134], $C_{3} \times D_{3}$ can be represented by

$$
s^{3}=t^{6}=e, t s t=s^{-1} .
$$

$G_{1}$ is formed by adjoining a generator $u$ with

$$
u^{3}=e, s u=u s, t u=u t .
$$

Let

$$
H_{1}=\left\{s, s^{-1}, t, t^{-1}, t s, t^{-1} s, t^{2} s, t^{-2} s^{-1}, u, u^{-1}, t u, t^{-1} u^{-1}\right\} .
$$

We assert that the Cayley graph $Y=X_{G_{1}, H_{1}}$ is a GRR of $G_{1}$. Let $Y_{e}$ denote the subgraph of $Y$ induced by the neighbors of $e$, namely the set $H_{1}$, and let $\varphi \in A_{e}(Y)$. The restriction of $\varphi$ to $Y_{e}$ belongs to $A\left(Y_{e}\right)$. One straightforwardly verifies that $Y_{e}$ has the form of Figure 1.

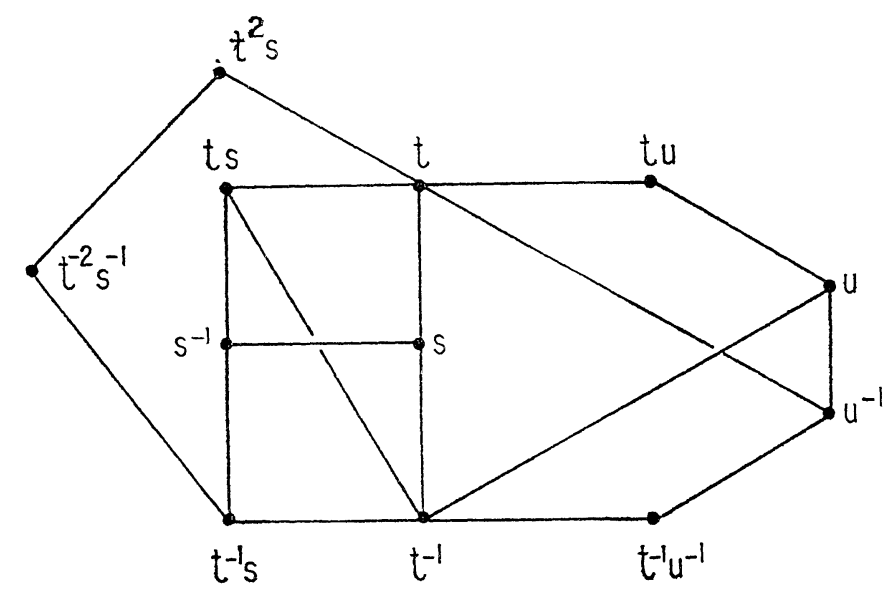

Figure 1. $Y_{e}$ when $G_{1}=C_{3} \times C_{3} \times D_{3}$

The two vertices whose valence in $Y_{e}$ is 5 are $t$ and $t^{-1}$, but only $t^{-1}$ has exactly one neighbor $t^{-1} u^{-1}$ of valence 2. Therefore, $\phi$ fixes 
both of these vertices and hence all vertices in the subgroup $\langle t, u\rangle$ generated by them. Then $\varphi$ fixes the second neighbor $t^{2} s$ of $t$ whose valence in $Y_{e}$ is 2 . But $G_{1}=\left\langle t^{2} s, t, u\right\rangle$, and so $\varphi$ is the identity and $Y$ is a GRR of $G_{1}$.

If $G=D_{6}$, we represent $G_{1}=C_{3} \times D_{6}$ by

$$
s^{6}=t^{2}=u^{3}=e, t s t=s^{-1}, s u=u s, t u=u t .
$$

Then $G_{1}$ is generated by

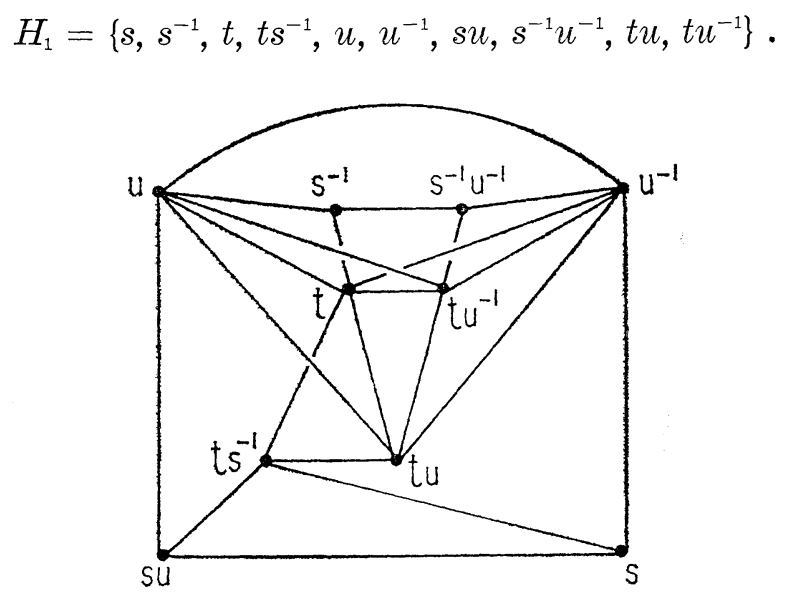

Figure 2. $Y_{e}$ when $G_{1}=C_{3} \times D_{6}$

Define $Y, Y_{e}$, and $\varphi$ as in the preceding paragraph. In this case (see Figure 2) $\varphi$ fixes $t s^{-1}$ since it is the only vertex whose valence in $Y_{e}$ is 4. Its only neighbor with valence 5 (in $Y_{e}$ ) is $t u$ and with valence 6 is $t$. But $\left\langle t s^{-1}, t, t u\right\rangle=G_{1}$, and so, as before $Y$ is a GRR of $G_{1}$.

REMARKS 1 . When $|G| \leqq 30$, the only cyclic extensions by $C_{3}$ which we have considered here are the direct products of $C_{3}$ with the various groups $G$ in Class I. Some of the other cases are readily dispensed with. For example, if $|G|=14,22$ or 26 , then $G_{1}$ is squarefree and is in Class I by Lemma 4.

There is a semi-direct product $G_{1}$ of $D_{6}$ by $C_{3}$ given by

$$
s^{6}=t^{2}=u^{3}=e, t s t=s^{-1}, s u=u s, u^{-1} t u=t s^{2} .
$$

If one lets $H_{1}=\left\{s, s^{-1}, t, t s^{-1}, u, u^{-1}, t u^{-1}, t s^{2} u\right\}$, then the Cayley graph $X_{G_{1}, H_{1}}$ is a GRR of $G_{1}$.

2. All cyclic extensions by $C_{3}$ of the two Class I groups $G$ of order 16 with exponent 8 are in Class I. Indeed in the light of (a), we need consider only the semi-direct product. First $G$ is generated by $s$ and $t$ where 


$$
s^{8}=t^{2}=e, t s t=t^{m}, m \equiv 3 \text { or } 7(\bmod 8) .
$$

Pick $c \in G_{1} \mid G$ such that $c^{3}=e$. The subgroup generated by $s$ is the only cyclic subgroup of order 8 and so is invariant. We have $c^{-1} s c=$ $s^{j}$ where $o\left(s^{j}\right)=8$. Thus $j^{3} \equiv 1(\bmod 8)$, and the only solution is $j \equiv$ $1(\bmod 8)$. But $G_{1}=G \times C_{3}$ is known to be in Class I by part (c).

3. Infinite graphs satisfy condition (b) in both Theorem 2 and Theorem 3. By virtue of Corollary 1A, these theorems and Corollary $2 \mathrm{~A}$ hold for infinite graphs.

ACKNOWLEDGEMENT. The authors wish to thank the referee for his careful reading and valuable suggestions, in particular for shortening the proof of Theorem 1 .

\section{REFERENCES}

1. C. Y. Chao, On a theorem of Sabidussi, Proc. Amer. Math. Soc., 15 (1964), 291292.

2. H. S. M. Coxeter and W. O. J. Moser, Generators and Relations for Discrete Groups, Springer-Verlag, Berlin, 1965.

3. Marshall Hall, Jr. and James K. Senior, The Groups of Order $2^{n}(n \leqq 6)$, Macmillan, New York, 1964.

4. Wilfried Imrich, Graphs with transitive Abelian automorphism group in Combinatorial Theory and Its Applications, Coll. Soc. János Bolyai 4, Balatonfüred, Hungary, (1969), 651-656.

5. Rudolf Kochendörffer, Lehrbuch der Gruppentheorie unter besonderer Berïcksichtigung der endlichen Gruppen, Leipzig, 1966.

6. Lewis A. Nowitz, On the non-existence of graphs with transitive generalized dicyclic groups, J. Combinatorial Theory, 4 (1968), 49-51.

7. Lewis A. Nowitz and Mark E. Watkins, Graphical regular representations of non-abelian, groups I, Canad. J. Math., 24 (1972), 993-1008.

8. — Graphical regular representations of non-abelian groups, II, Canad. J. Math., 24 (1972), 1009-1018.

9. Gert Sabidussi, On a class of fixed-point free graphs, Proc. Amer. Math. Soc., 9 (1958), 800-804.

10. - On vertex-transitive graphs, Monatsh. Math., 68 (1964), 426-438.

11. Mark E. Watkins, On the action of non-abelian groups on graphs, J. Combinatorial Theory, 11 (1971), 95-104.

12. - Graphical regular representations of alternating, symmetric, and miscellaneous small groups, Aequat. Math. 11 (1974), 40-50.

13. Mark E. Watkins and Lewis A. Nowitz, On graphical regular representations of direct products of groups, Monatsh. Math., 76 (1972), 168-171.

Received February 2, 1972 and in revised form October 1, 1973.

SYRACUSE UNIVERSITY

AND

Technische Hochschule, Vienna, Austria 



\section{PACIFIC JOURNAL OF MATHEMATICS}

\section{EDITORS}

RICHARD ARENS (Managing Editor)

University of California

Los Angeles, California 90024

\section{R. A. Beaumont \\ University of Washington \\ Seattle, Washington 98105}

\section{J. DugundJI}

Department of Mathematics

University of Southern California

Los Angeles, California 90007

D. Gilbarg and J. Milgram

Stanford University

Stanford, California 94305

\section{ASSOCIATE EDITORS}
E. F. BECKENBACH
B. H. NeumanN
F. WOLF
K. YOSHIDA

\section{SUPPORTING INSTITUTIONS}

UNIVERSITY OF BRITISH COLUMBIA
CALIFORNIA INSTITUTE OF TECHNOLOGY
UNIVERSITY OF CALIFORNIA
MONTANA STATE UNIVERSITY
UNIVERSITY OF NEVADA
NEW MEXICO STATE UNIVERSITY
OREGON STATE UNIVERSITY
UNIVERSITY OF OREGON
OSAKA UNIVERSITY

UNIVERSITY OF BRITISH COLUMBIA

UNIVERSITY OF CALIFORNIA

MONTANA STATE UNIVERSITY

NEW MEXICO STATE UNIVERSITY

OREGON STATE UNIVERSITY

OSAKA UNIVERSITY

\author{
UNIVERSITY OF SOUTHERN CALIFORNIA \\ STANFORD UNIVERSITY \\ UNIVERSITY OF TOKYO \\ UNIVERSITY OF UTAH \\ WASHINGTON STATE UNIVERSITY \\ UNIVERSITY OF WASHINGTON \\ AMERICAN MATHEMATICAL SOCIETY \\ NAVAL WEAPONS CENTER
}

The Supporting Institutions listed above contribute to the cost of publication of this Journal, but they are not owners or publishers and have no responsibility for its content or policies.

Mathematical papers intended for publication in the Pacific Journal of Mathematics should be in typed form or offset-reproduced, (not dittoed), double spaced with large margins. Underline Greek letters in red, German in green, and script in blue. The first paragraph or two must be capable of being used separately as a synopsis of the entire paper. Items of the bibliography should not be cited there unless absolutely necessary, in which case they must be identified by author and Journal, rather than by item number. Manuscripts, in triplicate, may be sent to any one of the editors. Please classify according to the scheme of Math. Reviews, Index to Vol. 39. All other communications should be addressed to the managing editor, or Elaine Barth, University of California, Los Angeles, California, 90024.

The Pacific Journal of Mathematics expects the author's institution to pay page charges, and reserves the right to delay publication for nonpayment of charges in case of financial emergency.

100 reprints are provided free for each article, only if page charges have been substantially paid. Additional copies may be obtained at cost in multiples of 50 .

The Pacific Journal of Mathematics is issued monthly as of January 1966. Regular subscription rate: $\$ 72.00$ a year (6 Vols., 12 issues). Special rate: $\$ 36.00$ a year to individual members of supporting institutions.

Subscriptions, orders for back numbers, and changes of address should be sent to Pacific Journal of Mathematics, 103 Highland Boulevard, Berkeley, California, 94708.

PUBLISHED BY PACIFIC JOURNAL OF MATHEMATICS, A NON-PROFIT CORPORATION

Printed at Kokusai Bunken Insatsusha (International Academic Printing Co., Ltd.), 270, 3-chome Totsuka-cho, Shinjuku-ku, Tokyo 160, Japan.

Copyright (C) 1973 by Pacific Journal of Mathematics Manufactured and first issued in Japan 


\section{Pacific Journal of Mathematics \\ Vol. 55, No. $2 \quad$ October, 1974}

Walter Allegretto, On the equivalence of two types of oscillation for elliptic

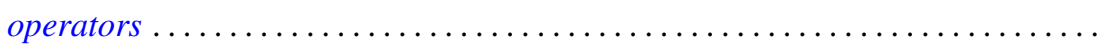

Edward Arthur Bertram, A density theorem on the number of conjugacy classes in

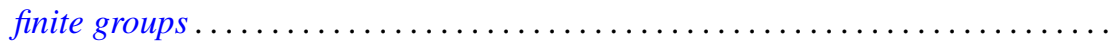

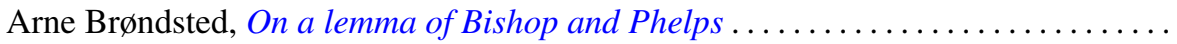

Jacob Burbea, Total positivity and reproducing kernels ..................

Ed Dubinsky, Linear Pincherle sequences . . . . . . . . . . . . . . . . . .

Benny Dan Evans, Cyclic amalgamations of residually finite groups .............

361

Barry J. Gardner and Patrick Noble Stewart, A "going down" theorem for certain

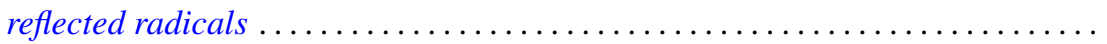

381

Jonathan Light Gross and Thomas William Tucker, Quotients of complete graphs:

revisiting the Heawood map-coloring problem ....................

Sav Roman Harasymiv, Groups of matrices acting on distribution spaces .........

Robert Winship Heath and David John Lutzer, Dugundji extension theorems for

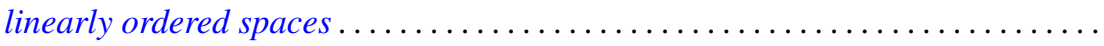

Chung-Wu Ho, Deforming p. l. homeomorphisms on a convex polygonal

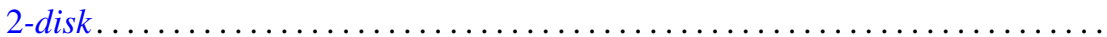

Richard Earl Hodel, Metrizability of topological spaces .................

Wilfried Imrich and Mark E. Watkins, On graphical regular representations of

cyclic extensions of groups .......................... 461

Jozef Krasinkiewicz, Remark on mappings not raising dimension of curves ..... . 479

Melven Robert Krom, Infinite games and special Baire space extensions . . . . . . 483

S. Leela, Stability of measure differential equations . . . . . . . . . . . . . . . . 489

M. H. Lim, Linear transformations on symmetric spaces . . . . . . . . . . . . . . . 499

Teng-Sun Liu, Arnoud C. M. van Rooij and Ju-Kwei Wang, On some group algebra modules related to Wiener's algebra $M_{1} \ldots \ldots \ldots \ldots \ldots \ldots \ldots \ldots \ldots \ldots \ldots$

Dale Wayne Myers, The back-and-forth isomorphism construction ............ 521

Donovan Harold Van Osdol, Extensions of sheaves of commutative algebras by

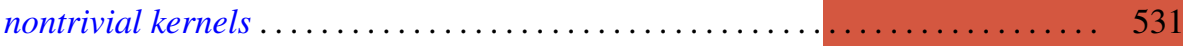

Alan Rahilly, Generalized Hall planes of even order ................... 543

Joylyn Newberry Reed, On completeness and semicompleteness of first countable

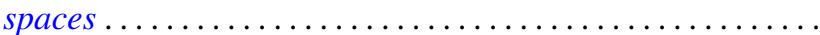

Alan Schwartz, Generalized convolutions and positive definite functions associated

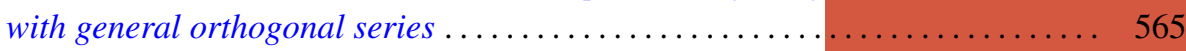

Thomas Jerome Scott, Monotonic permutations of chains . . . . . . . . . . . 583

Eivind Stensholt, An application of Steinberg's construction of twisted groups .... 595

Yasuji Takeuchi, On strongly radicial extensions . . . . ................. 619

William P. Ziemer, Some remarks on harmonic measure in space . . . . . . . . . . 629

John Grant, Corrections to: “Automorphisms definable by formulas” . . . . . . . . 639

Peter Michael Rosenthal, Corrections to: "On an inversion for the general

Mehler-Fock transform pair" ......................... 640

Carl Clifton Faith, Corrections to: "When are proper cyclics injective” . . . . . . 640 